\title{
Preparation and Structural Characterization of Dioxane-Coordinated Alkali Metal Bis(trimethylsilyl)amides
}

\author{
Frank T. Edelmann, Frank Pauer, Michael Wedler, and Dietmar Stalke* \\ Institut für Anorganische Chemie der Universität Göttingen, Tammannstrasse 4, \\ W-3400 Göttingen, Germany
}

Received March 20, 1992

Unsolvated $\mathrm{RbN}\left(\mathrm{SiMe}_{3}\right)_{2}(1)$ and $\mathrm{CsN}\left(\mathrm{SiMe}_{3}\right)_{2}(2)$ have been prepared from rubidium and cesium metal respectively and excess hexamethyldisilazane. The molecular structures of the polymeric dioxane adducts $\mathrm{NaN}\left(\mathrm{SiMe}_{3}\right)_{2} \cdot \mathrm{C}_{4} \mathrm{H}_{8} \mathrm{O}_{2}$ (3) and $\left[\mathrm{MN}\left(\mathrm{SiMe}_{3}\right)_{2} \cdot 1.5 \mathrm{C}_{4} \mathrm{H}_{8} \mathrm{O}_{2}\right] \cdot 0.5 \mathrm{C}_{4} \mathrm{H}_{8} \mathrm{O}_{2}(4, \mathrm{M}=\mathrm{Rb} ; 5, \mathrm{M}=\mathrm{Cs})$ have been determined by low-temperature single-crystal $\mathrm{X}$-ray diffraction.

\section{Introduction}

The bulky bis(trimethylsilyl)amido group, $-\mathrm{N}\left(\mathrm{SiMe}_{3}\right)_{2}$, plays an important role in the stabilization of low coordination numbers around main group and transition metal centers. ${ }^{1-3}$ Dicoordination of the transition metals manganese, iron, cobalt, and nickel was observed for the first time in solution ${ }^{4,5}$ and in the gas phase ${ }^{6,7}$ in the compounds $\mathrm{M}\left[\mathrm{N}\left(\mathrm{SiMe}_{3}\right)_{2}\right]_{2}(\mathrm{M}=\mathrm{Mn}, \mathrm{Fe}, \mathrm{Co}, \mathrm{Ni})$. The very unusual coordination number 3 for f-elements was realized in the lanthanide derivatives $\mathrm{Ln}\left[\mathrm{N}\left(\mathrm{SiMe}_{3}\right)_{2}\right]_{3}{ }^{8-10}$ The preparation of these metal bis(trimethylsilyl)amides usually involves treatment of the appropriate metal halides with either LiN$\left(\mathrm{SiMe}_{3}\right)_{2}$ or $\mathrm{NaN}\left(\mathrm{SiMe}_{3}\right)_{2}$. The structural chemistry of the lighter alkali metal derivatives $\mathrm{MN}\left(\mathrm{SiMe}_{3}\right)_{2}(\mathrm{M}=\mathrm{Li}, \mathrm{Na}, \mathrm{K})$ has already been studied in detail. ${ }^{11-17}$ In all three cases the base-free silyl amides have been structurally characterized. In addition, for $\mathbf{M}$ $=\mathrm{Li}, \mathrm{K}$ the molecular structures of several solvates containing diethyl ether, 12-crown-4, or dioxane have been investigated (Table I).

So far virtually nothing is known about bis(trimethylsilyl)amides containing the heavy alkali metals rubidium and cesium. We report here the synthesis of the hitherto unknown derivatives $\mathrm{RbN}\left(\mathrm{SiMe}_{3}\right)_{2}$ (1) and $\mathrm{CsN}\left(\mathrm{SiMe}_{3}\right)_{2}$ (2). Furthermore the molecular structures of three dioxane adducts, $\mathrm{NaN}\left(\mathrm{SiMe}_{3}\right)_{2}$. $2 \mathrm{C}_{4} \mathrm{H}_{8} \mathrm{O}_{2}(3)$ and $\left[\mathrm{MN}\left(\mathrm{SiMe}_{3}\right)_{2} \cdot 1.5 \mathrm{C}_{4} \mathrm{H}_{8} \mathrm{O}_{2}\right] \cdot 0.5 \mathrm{C}_{4} \mathrm{H}_{8} \mathrm{O}_{2}(4, \mathrm{M}$ $=\mathrm{Rb} ; \mathbf{5}, \mathrm{M}=\mathrm{Cs}$ ) have been determined by single-crystal $\mathrm{X}$-ray diffraction. These results allow a comparison of a series of dioxane-coordinated bis(trimethylsilyl)amides $\mathrm{MN}\left(\mathrm{SiMe}_{3}\right)_{2}$ (M $=\mathrm{Na}, \mathrm{K}, \mathrm{Rb}, \mathrm{Cs}$ ).

(1) Harris, D. H.; Lappert, M. F. J. Organomet. Chem. Libr. 1976, 2, 13

(2) Bradley, D. C.; Chisholm, M. H. Acc. Chem. Res. 1976, 9, 273.

(3) Power, P. P. Comments Inorg. Chem. 1989, 8, 177.

(4) Būrger, H.; Wannagat, U. Monatsh. Chem. 1963, 94, 1007.

(5) Bürger, H.: Wannagat, U. Monatsh, Chem. 1964, 95, 1099

(6) Bradley, D. C.; Fisher, K. J. J. Am. Chem. Soc. 1971, 93, 2058.

(7) Andersen, R. A.; Faegri, K., Jr.; Green, J. C.; Haaland, A.; Lappert, M. F.; Leung, W.-P.; Rypdal, K. Inorg. Chem. 1988, 27, 1782.

(8) Bradley, D. C.; Ghotra, J. S.; Hart, F. A. J. Chem. Soc., Dalton Trans. 1973, 1021 .

(9) Ghotra, J. S.; Hursthouse, M. B.; Welch, A. J. J. Chem. Soc., Chem. Commun. 1973, 669.

(10) Andersen, R. A.; Templeton, D. H.; Zalkin, A. Inorg. Chem. 1978, 17, 2317.

(11) Mootz, D.; Zinnius, A.; Böttcher, B. Angew. Chem. 1969, 81 , 398; Angew. Chem. Int. Ed. Engl. 1969, 8, 378.

(12) Fjeldberg, T.; Hitchcock, P. B.; Lappert, M. F.; Thorne, A. J. J. Chem Soc., Chem. Commun. 1984, 822.

(13) Engelhardt, L. M.; May, A. S.; Raston, C.; White, A. H. J. Chem. Soc., Dalton Trans. 1983, 1671

(14) Power, P. P.: Xiaojie, X. J. Chem. Soc, Chem. Commun. 1984, 358.

(15) Grüning, R.; Atwood, J. L. J. Organomet. Chem. 1977, 137, 101

(16) (a) Willard, P. G. Acta Crystallogr., C 1988, C44, 270. (b) Tesh, K. F.; Hanusa, T. P.; Huffman, J. C. Inorg. Chem. 1990, 29, 1584.

(17) Domingos, A. M.; Sheldrick, G. M. Acta Crystallogr., B 1974, B30, 517.

\section{Experimental Section}

All operations were carried out under an oxygen-free nitrogen atmosphere using standard Schlenk techniques. All solvents were rigorously dried by standard methods and freshly distilled from $\mathrm{Na}$ / benzophenone prior to use. NMR spectra were recorded on a Bruker AM 250 spectrometer. $\mathrm{NaN}\left(\mathrm{SiMe}_{3}\right)_{2}$ was obtained by reacting $\mathrm{NaNH}_{2}$ with $\mathrm{HN}\left(\mathrm{SiMe}_{3}\right)_{2}$ in toluene for $3 \mathrm{~d} .^{18}$

Synthesis of Rubidium Bis(trimethylsilyl)amide (1). A 1.0-g (12-mmol) sample of rubidium metal and an excess $(10 \mathrm{~mL}, 47 \mathrm{mmol})$ of hexamethyldisilazane were stirred at reflux temperature for $3 \mathrm{~d}$. During this time the alkali metal dissolved completely. The excess amine was removed in vacuo, and the residue was thoroughly dried to give $2.7 \mathrm{~g}$ (95\%) of a white amorphous powder. ${ }^{1} \mathrm{H}$ NMR $\left(\mathrm{C}_{6} \mathrm{D}_{6}\right): \delta 0.15$.

Synthesis of Cesium Bis(trimethylsilyl)amide (2). A 4.9-g (37-mmol) sample of cesium metal was suspended in a mixture of $100 \mathrm{~mL}$ of THF and $30 \mathrm{~mL}(142 \mathrm{mmol})$ of hexamethyldisilazane, and the mixture was refluxed for $60 \mathrm{~h}$. During this time the metal dissolved completely. The solution was filtered through a thin layer of Celite, and the clear filtrate was evaporated to dryness. The residue was washed with hexane and dried under vacuum to give $10.5 \mathrm{~g}(98 \%)$ of microcrystalline material. 'H NMR $\left(\mathrm{C}_{6} \mathrm{D}_{6}\right): \delta 0.19$.

Crystal Growth and $\mathrm{X}$-ray Structure Determinations. Suitable single crystals of 3, 4, and 5 were obtained by dissolving the base-free silylamides $\mathrm{MN}\left(\mathrm{SiMe}_{3}\right)_{2}(\mathrm{M}=\mathrm{Na}, \mathrm{Rb}, \mathrm{Cs})$ in a small amount of hot dioxane and slowly cooling the solutions to room temperature. The crystals were oil-coated shock-cooled at the tip of a fiber. The data were collected on a Stoe Siemens AED four-circle diffractometer using graphite-monochromated Mo $\mathrm{K} \alpha(\lambda=0.71073 \AA)$ radiation. The structure of 3 was solved by direct methods ${ }^{20}$ while the structures of 4 and 5 were solved by the Patterson method ${ }^{20}$ and refined by full-matrix least-squares techniques. ${ }^{20}$ In the case of 4 and 5 a semiempirical absorption correction was applied. All nonhydrogen atoms were refined anisotropically; all hydrogen atoms were found by difference Fourier syntheses and refined isotropically employing a riding model.

\section{Results and Discussion}

Preparation. $\mathrm{NaN}\left(\mathrm{SiMe}_{3}\right)_{2}$ was prepared from $\mathrm{NaNH}_{2}$ and $\mathrm{HN}\left(\mathrm{SiMe}_{3}\right)_{2}$ in toluene as previously described in the literature. ${ }^{19}$ The hitherto unknown rubidium and cesium bis(trimethylsilyl)amides were synthesized by reacting the metal with excess hexamethyldisilazane:

$$
\mathrm{M}+\mathrm{HN}\left(\mathrm{SiMe}_{3}\right)_{2} \rightarrow \mathrm{MN}\left(\mathrm{SiMe}_{3}\right)_{2}+1 / 2 \mathrm{H}_{2}
$$

The silylamides 1 and 2 are isolated in almost quantitative yields as white, moisture sensitive, microcrystalline solids. The diox-

(18) Pauer, F.; Stalke, D., J. Organomet. Chem., 1991, 418, 127.

(19) Brauer, G. Handbuch der Präparativen Anorganischen Chemie; Enke Verlag: Stuttgart, Germany, 1981; Vol. 2, p 712.

(20) Sheldrick, G. M. SHELX-86. Universităt Göttingen 1986. 
Table I. Selected Bond Lengths and Angles of Known Alkali Metal Bis(trimethylsilyl)amides

\begin{tabular}{|c|c|c|c|c|c|c|}
\hline compound & & $\mathrm{Si}-\mathrm{N}(\mathrm{pm})$ & $\mathrm{M}-\mathrm{N}(\mathrm{pm})$ & $\mathrm{Si}-\mathrm{N}-\mathrm{Si}(\mathrm{deg})$ & measurement temp (K) & ref \\
\hline$\left[\mathrm{LiN}\left(\mathrm{SiMe}_{3}\right)_{2}\right]_{3}$ & trimeric & 172.0 & 200.0 & 118.0 & 295 & 11 \\
\hline$\left[\mathrm{LiN}\left(\mathrm{SiMe}_{3}\right)_{2}\right]_{2}$ & dimeric & 171.2 & 199.0 & 129.8 & gas phase & 12 \\
\hline $\mathrm{LiN}\left(\mathrm{SiMe}_{3}\right)_{2} \cdot \mathrm{Et}_{2} \mathrm{O}$ & dimeric & 170.5 & 205.5 & 121.2 & $295^{2}$ & 13 \\
\hline $\mathrm{LiN}\left(\mathrm{SiMe}_{3}\right)_{2} \cdot 12$-crown-4 & monomeric & 168.1 & 196.5 & 123.5 & 140 & 14 \\
\hline $\mathrm{NaN}\left(\mathrm{SiMe}_{3}\right)_{2}$ & polymeric & $\begin{array}{l}169.4 \\
168.7\end{array}$ & $\begin{array}{l}235.2 \\
235.8\end{array}$ & 125.6 & 295 & 15 \\
\hline $\mathrm{NaN}\left(\mathrm{SiMe}_{3}\right)_{2} \cdot 2 \mathrm{C}_{4} \mathrm{H}_{8} \mathrm{O}_{2}$ & polymeric (linked monomers) & 167.2 & 238.0 & 139.8 & 153 & this work \\
\hline$\left[\mathrm{KN}\left(\mathrm{SiMe}_{3}\right)_{2}\right]_{2}$ & dimeric & 168.2 & $\begin{array}{l}277.0 \\
280.3\end{array}$ & 129.2 & 118 & 16 \\
\hline $\begin{array}{l}\mathrm{KN}\left(\mathrm{SiMe}_{3}\right)_{2} \cdot 2 \mathrm{C}_{4} \mathrm{H}_{8} \mathrm{O}_{2} \\
{\left[\mathrm{RbN}\left(\mathrm{SiMe}_{3}\right)_{2}\right]_{2} \cdot 3 \mathrm{C}_{4} \mathrm{H}_{8} \mathrm{O}_{2}}\end{array}$ & $\begin{array}{l}\text { polymeric (linked monomers) } \\
\text { polymeric (linked dimers) }\end{array}$ & $\begin{array}{l}164.0 \\
165.2 \\
167.7\end{array}$ & $\begin{array}{l}270.0 \\
294.6 \\
314.1\end{array}$ & $\begin{array}{l}136.2 \\
129.5\end{array}$ & $\begin{array}{l}295 \\
193\end{array}$ & $\begin{array}{l}17 \\
\text { this work }\end{array}$ \\
\hline$\left[\mathrm{CsN}\left(\mathrm{SiMe}_{3}\right)_{2}\right]_{2} \cdot 3 \mathrm{C}_{4} \mathrm{H}_{8} \mathrm{O}_{2}$ & polymeric (linked dimers) & $\begin{array}{l}167.2 \\
167.3\end{array}$ & $\begin{array}{l}306.7 \\
338.8\end{array}$ & 128.4 & 153 & this work \\
\hline
\end{tabular}

Table II. Crystallographic Data of 3,4 and 5

\begin{tabular}{|c|c|c|c|}
\hline & 3 & 4 & 5 \\
\hline $\begin{array}{l}\text { data collen at } T\left({ }^{\circ} \mathrm{C}\right) \\
\text { cryst size (mm) }\end{array}$ & $\begin{array}{l}-120 \\
0.4 \times 0.4 \times \\
0.6\end{array}$ & $\begin{array}{l}-80 \\
0.4 \times 0.4 \times \\
0.6\end{array}$ & $\begin{array}{l}-120 \\
0.4 \times 0.5 \times \\
\quad 0.7\end{array}$ \\
\hline space group & $\begin{array}{l}P 4,2,2 \\
1149,(1)\end{array}$ & $\begin{array}{l}P 2_{1} / n \\
11759(1)\end{array}$ & $\begin{array}{l}P 2_{1} / n \\
12597 \text { (2) }\end{array}$ \\
\hline$b(\mathrm{pm})$ & $1149.9(2)$ & $1123.3(2)$ & $1166.6(3)$ \\
\hline$c(\mathrm{pm})$ & $1579.5(1)$ & $1692.7(2)$ & $1520.0(3)$ \\
\hline $\begin{array}{l}\alpha(\mathrm{deg}): \\
\beta(\mathrm{deg}):\end{array}$ & & $\begin{array}{l}90 \\
93.91(1)\end{array}$ & $\begin{array}{l}90 \\
98.40(2)\end{array}$ \\
\hline$\gamma(\mathrm{deg}):$ & 90 & 90 & 90 \\
\hline cell vol $\left(\mathrm{nm}^{3}\right)$ : & 2.089 & 2.230 & 2.208 \\
\hline units per cell $Z$ : & 4 & 4 & 4 \\
\hline calcd density $D_{\mathrm{c}}\left(\mathrm{Mg} \mathrm{m}^{-3}\right)$ & 1.144 & 1.257 & 1.412 \\
\hline abs coeff $\mu\left(\mathrm{mm}^{-1}\right)$ : & $\begin{array}{l}0.20 \\
784\end{array}$ & $\begin{array}{l}2.28 \\
8.88\end{array}$ & 1.78 \\
\hline $\begin{array}{l}F(000) \\
2 \theta \text { range (deg): }\end{array}$ & $\begin{array}{l}784 \\
8-55\end{array}$ & $\begin{array}{l}888 \\
8-45\end{array}$ & $\begin{array}{l}960 \\
8-55\end{array}$ \\
\hline measd reflcns & 1516 & 4629 & 7533 \\
\hline no. of unique reflens & 1438 & 2896 & 5053 \\
\hline no. of obsd reflens & 1223 & 2223 & 4710 \\
\hline$p(F>p \sigma(F))$ & 3 & 3 & 3 \\
\hline$R$ & 0.0428 & 0.0703 & 0.0226 \\
\hline$R_{\mathrm{w}}$ & 0.0442 & 0.0738 & 0.0265 \\
\hline $\begin{array}{l}\text { weighting factor } g \\
\qquad\left(w=1 /\left(\sigma^{2}(F)+g F^{2}\right)\right.\end{array}$ & 0.0005 & 0.0010 & 0.0005 \\
\hline $\begin{array}{l}\text { no. of refined params } \\
\text { highest difference } \\
\text { peak }\left(e \AA^{-3}\right)\end{array}$ & $\begin{array}{l}101 \\
0.27\end{array}$ & $\begin{array}{l}199 \\
0.70\end{array}$ & $\begin{array}{l}301 \\
0.49\end{array}$ \\
\hline
\end{tabular}

ane adducts 3-5 were obtained by dissolving the base-free derivatives $\mathrm{MN}\left(\mathrm{SiMe}_{3}\right)_{2}$ in a minimum amount of hot dioxane. Slow cooling of these solutions to room temperature yielded $\mathrm{X}$ ray quality single crystals of the composition $\mathrm{NaN}\left(\mathrm{SiMe}_{3}\right)_{2} \cdot$ $2 \mathrm{C}_{4} \mathrm{H}_{8} \mathrm{O}_{2}$ (3) ( ${ }^{1} \mathrm{H}$ NMR $\left(\mathrm{C}_{6} \mathrm{D}_{6}\right) \delta 0.24\left(\mathrm{Si}\left(\mathrm{CH}_{3}\right)_{3}\right), 3.34$ $\left(\left[\mathrm{O}\left(\mathrm{CH}_{2}\right)_{2}\right]_{2}\right) ;{ }^{13} \mathrm{C}$ NMR $\left(\mathrm{C}_{6} \mathrm{D}_{6}\right) \delta 6.74\left(\mathrm{Si}\left(\mathrm{CH}_{3}\right)_{3}\right), 67.09$ $\left.\left(\left[\mathrm{O}\left(\mathrm{CH}_{2}\right)_{2}\right]_{2}\right) ;{ }^{29} \mathrm{Si} \mathrm{NMR} \delta-14.8\right),\left[\mathrm{RbN}\left(\mathrm{SiMe}_{3}\right)_{2}\right.$. $\left.1.5 \mathrm{C}_{4} \mathrm{H}_{8} \mathrm{O}_{2}\right] \cdot 0.5 \mathrm{C}_{4} \mathrm{H}_{8} \mathrm{O}_{2}(4)\left({ }^{1} \mathrm{H} \mathrm{NMR}\left(\mathrm{C}_{7} \mathrm{D}_{8}\right) \delta 0.15\left(\mathrm{Si}\left(\mathrm{CH}_{3}\right)_{3}\right)\right.$, $\left.3.32\left(\left[\mathrm{O}\left(\mathrm{CH}_{2}\right)_{2}\right]_{2}\right)\right)$, and $\left[\mathrm{CsN}\left(\mathrm{SiMe}_{3}\right)_{2} \cdot 1.5 \mathrm{C}_{4} \mathrm{H}_{8} \mathrm{O}_{2}\right] \cdot 0.5 \mathrm{C}_{4} \mathrm{H}_{8} \mathrm{O}_{2}$ (5) $\left({ }^{1} \mathrm{H} \mathrm{NMR}\left(\mathrm{C}_{6} \mathrm{D}_{6}\right) \delta 0.21\left(\mathrm{Si}\left(\mathrm{CH}_{3}\right)_{3}\right), 3.35\left(\left[\mathrm{O}\left(\mathrm{CH}_{2}\right)_{2}\right]_{2}\right) ;{ }^{3} \mathrm{C}\right.$ $\mathrm{NMR}\left(\mathrm{C}_{7} \mathrm{D}_{8}\right) \delta 7.18\left(\mathrm{Si}\left(\mathrm{CH}_{3}\right)_{3}\right), 67.13\left(\left[\mathrm{O}\left(\mathrm{CH}_{2}\right)_{2}\right]_{2}\right) ;{ }^{133} \mathrm{Cs} \mathrm{NMR}$ $\left.\left(\mathrm{C}_{4} \mathrm{D}_{8} \mathrm{O}\right) \delta 138\right)$.

Crystal Structures of 3, 4, and 5. The molecular structure of dioxane-solvated $\mathrm{KN}\left(\mathrm{SiMe}_{3}\right)_{2}$ has been known since $1973 .{ }^{17}$ In order to compare the structures of a whole series of dioxanecoordinated alkali metal bis(trimethylsilyl)amides, the crystal structures of 3-5 have been determined by single-crystal X-ray diffraction. The crystallographic data for 3-5 are given in Table II, atomic coordinates and equivalent isotropic displacement parameters in Tables III-V. Table VI contains selected bond lengths and bond angles.

Polymeric $\mathrm{NaN}\left(\mathrm{SiMe}_{3}\right)_{2} \cdot 2 \mathrm{C}_{4} \mathrm{H}_{8} \mathrm{O}_{2}$ (3) (Figure 1) is isostructural with the corresponding potassium derivative. The central sodium a tom is coordinated by the nitrogen atom of the $\mathrm{N}\left(\mathrm{SiMe}_{3}\right)_{2}$ ligand and four oxygen atoms, resulting in a distorted trigonalbipyramidal coordination geometry. The nitrogen and two oxygen atoms are in the equatorial plane, while two oxygen atoms occupy

Table III. Atomic Coordinates $\left(\times 10^{4}\right)$ and Equivalent Isotropic Displacement Parameters $\left(\AA^{2} \times 10^{3}\right)$ of $3^{a}$

\begin{tabular}{lllll}
\hline & $x$ & $y$ & \multicolumn{1}{c}{$z$} & $U(\mathrm{eq})$ \\
\hline $\mathrm{Na}(1)$ & $6083(1)$ & $3917(1)$ & 2500 & $25(1)$ \\
$\mathrm{N}(1)$ & $4620(2)$ & $5380(2)$ & 2500 & $22(1)$ \\
$\mathrm{Si}(1)$ & $4799(1)$ & $6416(1)$ & $3232(1)$ & $25(1)$ \\
$\mathrm{C}(11)$ & $3714(3)$ & $6394(3)$ & $4122(2)$ & $44(1)$ \\
$\mathrm{C}(12)$ & $4757(4)$ & $7947(3)$ & $2813(2)$ & $48(1)$ \\
$\mathrm{C}(13)$ & $6252(3)$ & $6229(3)$ & $3753(2)$ & $43(1)$ \\
$\mathrm{O}(1)$ & $7838(2)$ & $3488(2)$ & $3282(1)$ & $28(1)$ \\
$\mathrm{C}(1)$ & $8656(3)$ & $4215(3)$ & $3707(2)$ & $32(1)$ \\
$\mathrm{C}(2)$ & $7820(3)$ & $2366(3)$ & $3681(2)$ & $26(1)$ \\
$\mathrm{O}(2)$ & $7557(2)$ & $5179(2)$ & $1630(1)$ & $29(1)$ \\
$\mathrm{C}(3)$ & $6827(2)$ & $5993(3)$ & $1187(2)$ & $26(1)$ \\
$\mathrm{C}(4)$ & $8671(3)$ & $5145(3)$ & $1228(2)$ & $33(1)$
\end{tabular}

a $U\left(\right.$ eq) is defined as one-third of the trace of the orthogonalized $\mathbf{U}_{i j}$ tensor.

Table IV. Atomic Coordinates $\left(\times 10^{4}\right)$ and Equivalent Isotropic Displacement Parameters $\left(\AA^{2} \times 10^{3}\right)$ of $4^{0}$

\begin{tabular}{lrlrr}
\hline & $x$ & $y$ & $z$ & $U($ eq $)$ \\
\hline $\mathrm{Rb}(1)$ & $-1401(1)$ & $4811(1)$ & $5692(1)$ & $44(1)$ \\
$\mathrm{N}(1)$ & $657(5)$ & $6305(5)$ & $5894(3)$ & $47(2)$ \\
$\mathrm{Si}(1)$ & $1575(2)$ & $5739(2)$ & $6575(1)$ & $50(1)$ \\
$\mathrm{C}(11)$ & $1084(9)$ & $5773(13)$ & $7613(5)$ & $115(6)$ \\
$\mathrm{C}(12)$ & $3041(7)$ & $6443(8)$ & $6650(6)$ & $80(4)$ \\
$\mathrm{C}(13)$ & $1860(9)$ & $4124(8)$ & $6382(6)$ & $82(4)$ \\
$\mathrm{Si}(2)$ & $351(2)$ & $7734(2)$ & $5684(1)$ & $51(1)$ \\
$\mathrm{C}(21)$ & $-1225(7)$ & $7984(8)$ & $5435(6)$ & $76(4)$ \\
$\mathrm{C}(22)$ & $750(8)$ & $8851(8)$ & $6488(6)$ & $77(4)$ \\
$\mathrm{C}(23)$ & $1079(11)$ & $8288(9)$ & $4795(6)$ & $101(5)$ \\
$\mathrm{O}(1)$ & $-2607(5)$ & $6276(5)$ & $7078(3)$ & $65(2)$ \\
$\mathrm{O}(2)$ & $-1646(6)$ & $3226(5)$ & $7050(4)$ & $76(3)$ \\
$\mathrm{C}(1)$ & $-3669(8)$ & $6268(8)$ & $7406(6)$ & $70(4)$ \\
$\mathrm{C}(2)$ & $-858(8)$ & $2508(8)$ & $7536(6)$ & $76(4)$ \\
$\mathrm{C}(3)$ & $-2715(8)$ & $3237(9)$ & $7377(7)$ & $80(4)$ \\
$\mathrm{C}(4)$ & $-1824(9)$ & $6987(9)$ & $7555(7)$ & $89(5)$ \\
$\mathrm{O}(3)$ & $-3926(5)$ & $4860(5)$ & $5417(4)$ & $65(2)$ \\
$\mathrm{C}(5)$ & $-4827(7)$ & $4042(8)$ & $5514(6)$ & $65(4)$ \\
$\mathrm{C}(6)$ & $-4396(7)$ & $6017(8)$ & $5224(6)$ & $70(4)$ \\
$\mathrm{O}(4)$ & $906(16)$ & $5634(19)$ & $-167(10)$ & $245(10)$ \\
$\mathrm{C}(7)$ & $-11(15)$ & $6164(11)$ & $-4(11)$ & $156(9)$ \\
$\mathrm{C}(8)$ & $624(17)$ & $4370(15)$ & $-463(10)$ & $150(9)$
\end{tabular}

${ }^{a} U(\mathrm{eq})$ is defined as one-third of the trace of the orthogonalized $\mathbf{U}_{i j}$ tensor.

the axial positions. The Si-N bond length (average $167.8 \mathrm{pm}$ ) is longer and the $\mathrm{Si}-\mathrm{N}-\mathrm{Si}$ angle $\left(130.6^{\circ}\right)$ is smaller than in the corresponding potassium derivative $\mathrm{KN}\left(\mathrm{SiMe}_{3}\right)_{2} \cdot 2 \mathrm{C}_{4} \mathrm{H}_{8} \mathrm{O}_{2}(164.0$ $\mathrm{pm}$ and $136.3^{\circ}$ respectively). ${ }^{17}$ This may be attributed to a lesser degree of ionic character in the sodium compound. In the unsolvated $\mathrm{NaN}\left(\mathrm{SiMe}_{3}\right)_{2}$ the average $\mathrm{Si}-\mathrm{N}$ distance is $169.0 \mathrm{pm}$ and the $\mathrm{Si}-\mathrm{N}-\mathrm{Si}$ angle is $125.6^{\circ} . .^{15}$ As with most compounds in which more than one silyl group is attached to nitrogen, the nitrogen atom adopts a planar geometry. The monomeric units are linked via 1,4-dioxane bridges to give an overall polymeric structure.

$\left[\mathrm{RbN}\left(\mathrm{SiMe}_{3}\right)_{2}\right]_{2} \cdot 3 \mathrm{C}_{4} \mathrm{H}_{8} \mathrm{O}_{2}(4)$ and $\left[\mathrm{CsN}\left(\mathrm{SiMe}_{3}\right)_{2}\right]_{2} \cdot 3 \mathrm{C}_{4} \mathrm{H}_{8} \mathrm{O}_{2}$ 
Table V. Atomic Coordinates $\left(\times 10^{4}\right)$ and Equivalent Isotropic Displacement Parameters $\left(\AA^{2} \times 10^{3}\right)$ of $5^{a}$

\begin{tabular}{lrlll}
\hline & \multicolumn{1}{c}{$\boldsymbol{x}$} & $y$ & $z$ & $U(\mathrm{eq})$ \\
\hline $\mathrm{Cs}(1)$ & $-1367(1)$ & $4839(1)$ & $5644(1)$ & $25(1)$ \\
$\mathrm{N}(1)$ & $508(1)$ & $6519(1)$ & $6091(1)$ & $24(1)$ \\
$\mathrm{Si}(1)$ & $1428(1)$ & $6088(1)$ & $6932(1)$ & $24(1)$ \\
$\mathrm{C}(11)$ & $1392(2)$ & $6793(2)$ & $8043(2)$ & $40(1)$ \\
$\mathrm{C}(12)$ & $2862(2)$ & $6283(2)$ & $6737(2)$ & $42(1)$ \\
$\mathrm{C}(13)$ & $1276(2)$ & $4503(2)$ & $7134(2)$ & $44(1)$ \\
$\mathrm{Si}(2)$ & $270(1)$ & $7847(1)$ & $5695(1)$ & $26(1)$ \\
$\mathrm{C}(21)$ & $-1108(2)$ & $7948(2)$ & $5024(2)$ & $50(1)$ \\
$\mathrm{C}(22)$ & $338(2)$ & $9030(2)$ & $6547(2)$ & $42(1)$ \\
$\mathrm{C}(23)$ & $1241(3)$ & $8334(2)$ & $4932(2)$ & $57(1)$ \\
$\mathrm{O}(1)$ & $-2522(1)$ & $6242(1)$ & $7012(1)$ & $36(1)$ \\
$\mathrm{O}(2)$ & $-1659(1)$ & $3174(1)$ & $7174(1)$ & $41(1)$ \\
$\mathrm{C}(1)$ & $-3525(2)$ & $6200(2)$ & $7344(2)$ & $34(1)$ \\
$\mathrm{C}(2)$ & $-967(2)$ & $2367(2)$ & $7687(2)$ & $41(1)$ \\
$\mathrm{C}(3)$ & $-2668(2)$ & $3210(2)$ & $7499(2)$ & $41(1)$ \\
$\mathrm{C}(4)$ & $-1824(2)$ & $7041(2)$ & $7522(2)$ & $39(1)$ \\
$\mathrm{O}(3)$ & $-3900(1)$ & $4822(1)$ & $5307(1)$ & $39(1)$ \\
$\mathrm{C}(5)$ & $-4610(2)$ & $3879(2)$ & $5082(2)$ & $38(1)$ \\
$\mathrm{C}(6)$ & $-4264(2)$ & $5801(2)$ & $4787(2)$ & $42(1)$ \\
$\mathrm{O}(4)$ & $-291(2)$ & $3843(1)$ & $9837(1)$ & $50(1)$ \\
$\mathrm{C}(7)$ & $-1067(2)$ & $4713(3)$ & $9796(2)$ & $56(1)$ \\
$\mathrm{C}(8)$ & $676(3)$ & $4314(3)$ & $9607(3)$ & $63(1)$ \\
& & & &
\end{tabular}

a $U(\mathrm{eq})$ is defined as one-third of the trace of the orthogonalized $\mathrm{U}_{i j}$ tensor.

Table VI. Selected Bond Lengths (pm) and Bond Angles (deg) for 3,4 , and 5

\begin{tabular}{|c|c|c|c|}
\hline \multicolumn{4}{|c|}{ Compound 3} \\
\hline $\begin{array}{l}\mathrm{Na}(1)-\mathrm{N}(1) \\
\mathrm{Na}(1)-\mathrm{O}(1) \\
\mathrm{Na}(1)-\mathrm{O}(2) \\
\mathrm{Si}(1)-\mathrm{N}(1)\end{array}$ & $\begin{array}{l}238.0(1) \\
241.7(2) \\
262.1(2) \\
167.3(2)\end{array}$ & $\begin{array}{l}\mathrm{Si}(1)-\mathbf{N}(1)-\mathrm{Si}(1 \mathrm{a}) \\
\mathrm{Si}(1)-\mathbf{N}(1)-\mathrm{Na}(1)\end{array}$ & $\begin{array}{l}139.8(1) \\
114.6(1)\end{array}$ \\
\hline $\begin{array}{l}\mathbf{R b}(1)-N(1) \\
\mathrm{Rb}(1)-\mathrm{N}(1 \mathrm{a}) \\
\mathrm{Rb}(1)-\mathrm{O}(1) \\
\mathrm{Rb}(1)-\mathrm{O}(2) \\
\mathrm{Rb}(1)-\mathrm{O}(3) \\
\mathrm{Si}(1)-\mathrm{N}(1) \\
\mathrm{Si}(2)-\mathrm{N}(1) \\
\mathrm{Rb}(1) \cdots \mathrm{Rb}(1 \mathrm{a})\end{array}$ & $\begin{array}{l}\text { Co } \\
294.6(6) \\
314.1(6) \\
326.6(6) \\
293.4(6) \\
297.4(6) \\
165.2(6) \\
167.7(6) \\
419.4(2)\end{array}$ & $\begin{array}{l}\text { pound } 4 \\
\mathrm{Si}(1)-\mathrm{N}(1)-\mathrm{Si}(2) \\
\mathrm{N}(1)-\mathrm{Rb}(1)-\mathrm{N}(1 \mathrm{a}) \\
\mathrm{Rb}(1)-\mathrm{N}(1)-\mathrm{Rb}(1 \mathrm{a})\end{array}$ & $\begin{array}{r}129.5(4) \\
93.0(2) \\
87.0(2)\end{array}$ \\
\hline $\begin{array}{l}\mathrm{Cs}(1)-N(1) \\
\mathrm{Cs}(1)-\mathrm{N}(1 \mathrm{a}) \\
\mathrm{Cs}(1)-\mathrm{O}(1) \\
\mathrm{Cs}(1)-\mathrm{O}(2) \\
\mathrm{Cs}(1)-\mathrm{O}(3) \\
\mathrm{Si}(1)-\mathrm{N}(1) \\
\mathrm{Si}(2)-\mathrm{N}(1) \\
\mathrm{Cs}(1) \ldots \mathrm{Cs}(1 \mathrm{a})\end{array}$ & $\begin{array}{l}\text { Co } \\
306.7(1) \\
338.8(2) \\
316.1(2) \\
309.3(2) \\
315.3(1) \\
167.2(2) \\
167.3(2) \\
421.9(1)\end{array}$ & $\begin{array}{l}\text { ppound } 5 \\
\mathrm{Si}(1)-N(1)-S i(2) \\
N(1)-\operatorname{Cs}(1)-N(1 \mathrm{a}) \\
\mathrm{Cs}(19)-N(1)-\operatorname{Cs}(1 \mathrm{a})\end{array}$ & $\begin{array}{r}128.4(1) \\
98.5(1) \\
81.5(1)\end{array}$ \\
\hline
\end{tabular}

(5) also form a pair of isostructural compounds. Figures 2 and 3 show the structures of the dimeric subunits $\mathrm{MN}\left(\mathrm{SiMe}_{3}\right)_{2}$. $3 \mathrm{C}_{4} \mathrm{H}_{8} \mathrm{O}_{2}$. The metal cations are coordinated by two nitrogen and three oxygen atoms. This results in a distorted tetragonalpyramidal coordination geometry around the metal centers. A center of inversion lies in the middle of the central four-membered $\mathrm{M}_{2} \mathrm{~N}_{2}$ ring. The metal-nitrogen bonds within the four-membered ring are not equivalent. The difference in the $\mathrm{Rb}-\mathrm{N}$ bond lengths in $4(\mathrm{Rb}(1)-\mathrm{N}(1)=294.6(6) \mathrm{pm} ; \mathrm{Rb}(1)-\mathrm{N}(1 \mathrm{a})=314.1(6)$ $\mathrm{pm})$ is $19.5 \mathrm{pm}$; that of the Cs-N distances in $5(\mathrm{Cs}(1)-\mathrm{N}(1)=$ $306.7(1) \mathrm{pm} ; \mathrm{Cs}(1)-\mathrm{N}(1 \mathrm{a})=338.8 \mathrm{pm})(2)$ is even larger (32.1 $\mathrm{pm})$. In 4 the $\mathrm{N}(1)$ is as much as $32.3 \mathrm{pm}$ above the $\mathrm{Si}(1), \mathrm{Si}(2)$, $\mathrm{Rb}$ (1) plane. The $\mathrm{Rb}(1)-\mathrm{N}(1)$ vector forms an angle of $123.4^{\circ}$ with the $\mathrm{Si}(1), \mathrm{Si}(2), \mathrm{N}(1)$ plane. This means the plane of the ligand almost bisects the $\mathrm{Rb}(1)-\mathrm{N}(1)-\mathrm{Rb}(1 \mathrm{a})$ bond angle. In 5 the $\mathrm{N}(1)$ atom is only $19.5 \mathrm{pm}$ above the $\mathrm{Si}(1), \mathrm{Si}(2), \mathrm{Cs}(1)$ plane, indicating nearly planar environment of the nitrogen atom. The Cs(1)-N(1) vector forms an angle of $160.6^{\circ}$ with the $-\mathrm{N}\left(\mathrm{SiMe}_{3}\right)_{2}$ ligand plane. That means despite the steric hinderence the ligand is almost entirely bended toward the sym-

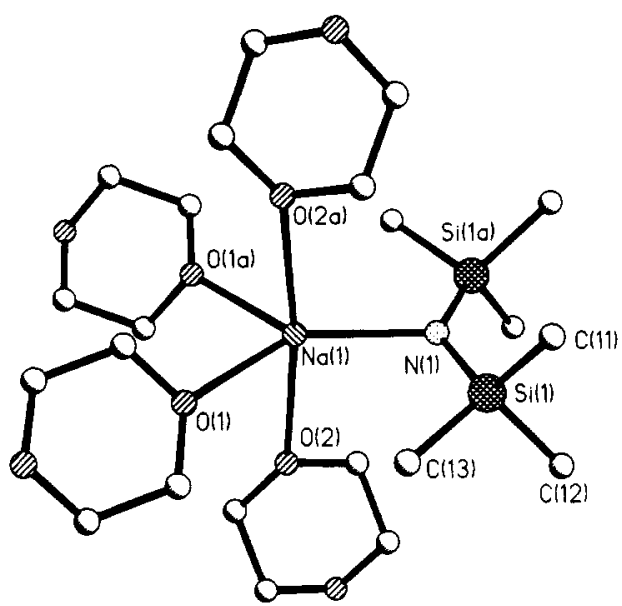

Figure 1. Structure of $\mathrm{NaN}\left(\mathrm{SiMe}_{3}\right)_{2} \cdot 2 \mathrm{C}_{4} \mathrm{H}_{8} \mathrm{O}_{2}(3)$ in the solid state.

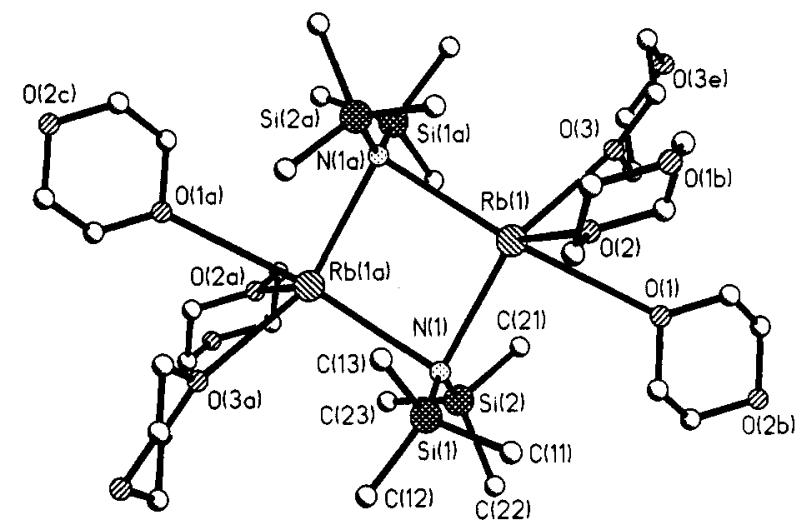

Figure 2. Structure of $\mathrm{RbN}\left(\mathrm{SiMe}_{3}\right)_{2} \cdot 1.5 \mathrm{C}_{4} \mathrm{H}_{8} \mathrm{O}_{2}(4)$ in the solid state. The uncoordinated lattice solvent dioxane is omitted.

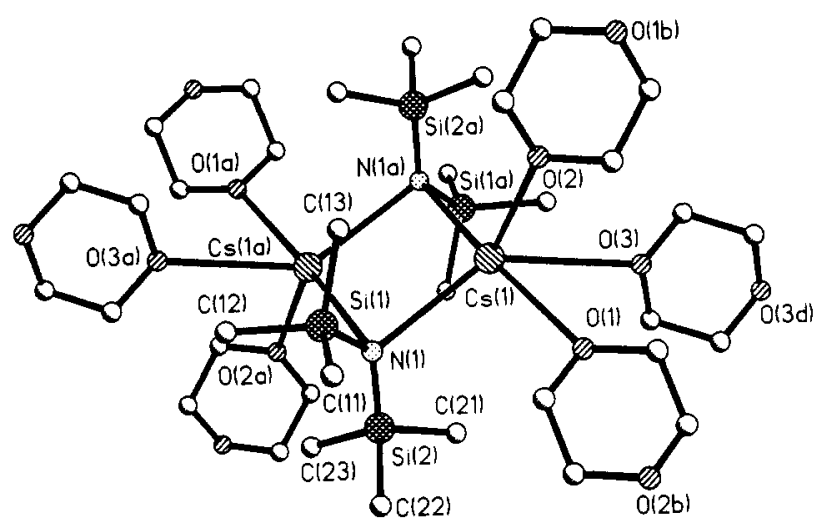

Figure 3. Structure of $\mathrm{CsN}\left(\mathrm{SiMe}_{3}\right)_{2} \cdot 1.5 \mathrm{C}_{4} \mathrm{H}_{8} \mathrm{O}_{2}(5)$ in the solid state. The uncoordinated lattice solvent dioxane is omitted.

metry-related metal atom $\mathrm{Cs}(1 \mathrm{a})$. This geometrical arrangement together with the asymmetric bridging and the planar environment of $N(1)$ indicates that the nitrogen atom is not $\mathrm{sp}^{3}$ hybridized. We suggest that the nitrogen atom in 5 is $\mathrm{sp}^{2}$ hybridized and the lone pair is located in an unhybridized p atom orbital. This orbital is perpendicular to the $\mathrm{Si}(1), \mathrm{Si}(2), \mathrm{N}(1), \mathrm{Cs}(1)$ plane and directed toward the second metal Cs(1a), forming the longer distance of $338.8(2) \mathrm{pm}$, whereas the shorter bond is realized by the $\mathrm{sp}^{2}$ orbital directed toward Cs(1) (Figure 4).

On the other hand we think that the bonding situation in the $\mathbf{R b}-\mathbf{N}$ four-membered ring is described more adequately by a $\mathrm{sp}^{3}$-hybridized nitrogen atom. The $\mathrm{Rb}-\mathrm{N}$ distances differ only by $19.5 \mathrm{pm}$ and the $-\mathrm{N}\left(\mathrm{SiMe}_{3}\right)_{2}$ ligand plane bisects the $\mathrm{Rb}$ (1)-N-Rb(1a) angle (Figure 4). However, both descriptions may be too simple minded, and the truth is probably somewhere in between. 

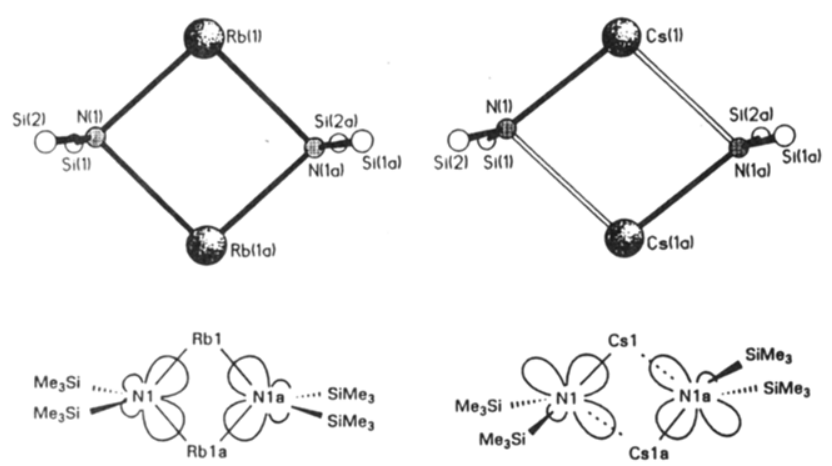

Figure 4. Bonding situation in the $\mathrm{Rb}_{2} \mathrm{~N}_{2}$ and the $\mathrm{Cs}_{2} \mathrm{~N}_{2}$ four-membered ring. The nitrogen atom in the first ring is $\mathbf{s p}^{3}$ hybridized and is $\mathbf{s p}^{2}$ hybridized in the latter.
The Rb-N and Cs- $\mathrm{N}$ bond lengths in 4 and 5 are in good agreement with the distances in the central $\mathrm{N}_{2} \mathbf{M}_{2}$-ring of $\left[\mathrm{PhS}\left(\mathrm{NSiMe}_{3}\right)_{2} \mathrm{Rb} \cdot \mathrm{THF}\right]_{2}{ }^{18}$ and $\left[\mathrm{PhS}\left(\mathrm{NSiMe}_{3}\right)_{2} \mathrm{Cs} \cdot \mathrm{THF}\right]_{2}{ }^{18}$ Furthermore, each coordinated dioxane ligand bridges two metal centers, thus connecting the dimers to give an overall polymeric structure.

Acknowledgment. This work was financially supported by the Deutsche Forschungsgemeinschaft and the Fonds der Chemischen Industrie. We are especially grateful to Prof. Dr. H. W. Roesky and Prof. G. M. Sheldrick for their support.

Supplementary Material Available: Listings of crystal and diffractometer data, atomic coordinates, bond lengths, bond angles, and anisotropic displacement coefficients (18 pages). Ordering information is given on any current masthead page. 